\title{
Análisis del proceso de adopción de sistemas de gestión de ecodiseńo en cooperativas del sector de bienes de equipo
}

\author{
Beñat Landeta-Manzano \\ Profesor adjunto del departamento de Organización de Empresas \\ Germán Arana-Landín \\ Profesor Titular de Universidad del departamento de Organización de Empresas \\ Patxi Ruíz de Arbulo \\ Profesor Agregado del departamento de Organización de Empresas \\ Pablo Díaz de Basurto \\ Catedrático de Universidad del departamento de Organización de Empresas
}

DOI: $10.1387 /$ reves. 17536

Fecha de entrada: 30/10/2015

Fecha de aceptación: 14/12/2015

Sumario: 1. Introducción. 2. Metodología. 3. Análisis de casos. 4. Conclusiones. 5. Referencias bibliográficas.

\section{Resumen:}

La norma ISO 14006 de diseño ecológico o ecodiseño se creó en 2011 con el fin de complementar el estándar ISO 14001 de gestión medioambiental. Aunque hay una gran cantidad de referencias en la literatura que analizan el proceso de adopción de la ISO 14001, el estándar de ecodiseño no ha recibido tanta atención dado que no ha tenido la difusión esperada. A este respecto, con el fin de contribuir de paliar esta carencia, el objetivo de este artículo es realizar un análisis exploratorio sobre el proceso de adopción e integración de la ISO 14006 en la gestión ambiental de dos cooperativas vascas que han sido pioneras en hacerlo a nivel estatal. Tras el análisis de los principales motivos que han llevado a las empresas a adoptar la estándar ISO 14006, la investigación se ha centrado en el análisis del impacto de dicha adopción, tanto a nivel operativo como en términos de resultados, en las empresas. Con todo ello, sean extraído una serie de conclusiones que se esperan pueda ser de interés tanto para las empresas y para y a otros grupos 
de interés, tales como los decisores públicos que tienen como fin establecer políticas orientadas a la mejora de la eficiencia en la gestión medioambiental y de los recursos.

\title{
Palabras clave:
}

Ecoinnovación, Gestión ambiental, Desarrollo sostenible, Bienes de equipo, Cooperativas

\section{Laburpena:}

Diseinu ekologiko edo ekodiseinu ISO 14006 araua 2011 an argitaratu zen, ingurugiroa kudeatzeko ISO 14001 araua osatzearren. Literaturan, ISO 14001 enpresetan ezarri eta ziurtatze prozesuari buruzko erreferentzia asko daude, ostera ISO 14006 arauaren inguruko erreferentziak gutxi dira, bere zabalkundea espero zena baino eskasagoa izan baita. Horregatik, artikulu honen asmoa bi euskal kooperatiba enpresen kudeaketa sisteman ISO 14006-ren ezarpen eta integrazio prozesuari buruzko azterketa erakustea da. Bi enpresa hauek aitzindariak dira arlo honetan. Hargatik, prozesua martxan jartzeko erabakiaren zergatiak, prozesua beraren nondik norakoak ISO 14006-ren araberako ziurtagiria erdietsi arte, eta lortutako emaitza ekonomikoak aztertu ditugu. Bereziki, ISO 14006-ren ezarpen eta integrazio prozesuaren eragina maila operatibo eta ekonomikoan arreta jarri dugu. Honi esker, ISO 14006 arauaren araberako kudeaketa sistema ezartzeko asmoa daukaten enpresentzat eta bere inguruko interes-taldeentzat, esaterako, ingurugiroaren eta baliabideen kudeaketa eraginkortasuna hobetzeko politika publikoak ezartzen dituzten erakundeentzat, interesgarriak izan daitezkeen ondorioak aurkezten ditugu.

\section{Gako-hitzak:}

Eko-berrikuntza, Ingurugiroaren kudeaketa, Garapen jasangarria, Kapital-ondasunak, Kooperatibak

\begin{abstract}
:
The ISO 14006 standard eco-design or eco-design was created in 2011 to complement the successful ISO 14001 environmental management standard. There are a lot of references in the literature to analyze the process of adoption of ISO 14001; however, the eco-design standard has not received much attention. In this regard, in order to contribute to alleviate this deficiency, the aim of this article is to make an exploratory analysis on the process of adoption and integration of ISO 14006 in Management-Environmental Spanish cooperative enterprises that have pioneered do it statewide. After analysis of the main reasons that led companies to adopt the standard ISO 14006, research has focused on analyzing the impact of the adoption, both operationally
\end{abstract}


ANÁLISIS DEL PROCESO DE ADOPCIÓN DE SISTEMAS DE GESTIÓN DE ECODISEÑO...

and in terms of outcomes, in enterprises. In all, they are drawn a number of conclusions that are expected to be of interest both for companies and for other stakeholders, such as public decision makers who aim to establish policies aimed at improving efficiency in environmental management and resources.

\section{Keywords:}

Eco-innovation, Environmental management, Sustainable development, Capital goods, Cooperatives

Claves Econlit:

M420, Q560, O310

\section{Introducción}

La agrupación sectorial de bienes de equipo es clave en la industria y debe dar respuesta a la elevada competitividad de los mercados, tanto a nivel nacional como internacional (Ramos, 2012). Lleva muchos años trabajando para mejorar el comportamiento ambiental de sus actividades, tratando de reducir los impactos ambientales globales generados en sus procesos de fabricación (Ramos, 2012). En los últimos años una creciente preocupación ambiental entre los clientes ha impulsado soluciones que han permitido mejorar también los impactos ambientales generados a lo largo de todo el ciclo de vida de los productos (Arana y Heras, 2011; Arana et al., 2012).

Por otro lado, se trata de un sector en el que la tecnología se iguala y difunde cada vez con mayor rapidez, por lo que el factor tecnológico pierde peso como factor de competencia para lograr la diferenciación de la empresa en el mercado (Ramos, 2012). En este contexto el ecodiseńo se plantea como una herramienta de innovación que permite a las empresas mejorar su competitividad y ofrecer unos productos más respetuosos con el medio ambiente (Arana et al., 2012; Landeta et al. 2015; Landeta et al, 2016).

En este ámbito precisamente las cooperativas de la Comunidad Autónoma del País Vasco han sido pioneras. Concretamente, a finales de los 90, impulsados por la Sociedad de Gestión Ambiental del Gobierno Vasco, Ihobe, un grupo formado por cuatro empresas industriales: Daisalux, Ofita, y Fagor electrodomésticos, sección mini-domésticos y sección lavadoras, junto con dos ingenierías de diseño Diara, perteneciente a Corporación Mondragón, y Tres D Innovación y Diseño Integral, desarrollaron un proyecto piloto en materia de Gestión de Ecodiseño. A su finalización, dados los buenos resultados del proyecto, trasladaron a la Asociación Española de Estándarlización y Certificación $(A E N O R)$ la necesidad de contar una estándar en la que se describiese un modelo de gestión del ecodiseño, que facilitara la incorporación de la variable am- 
biental en el diseño de productos y/o servicios (Alonso, 2011). Así, se pasaría de observar las acciones de mejora ambiental centradas fundamentalmente en la reducción de impactos asociados a los procesos de producción, a una visión completa del ciclo de vida del producto (Fernandez, 2012).

A la vista de los acontecimientos, AENOR, organismo responsable del desarrollo y difusión de las estándars técnicas en España, creó un comité técnico de estándarlización con el fin de elaborar una estándar UNE sobre ecodiseño, presidido por Ihobe, que contó con la colaboración de expertos de las empresas que habían participado en el proyecto Piloto (Alonso, 2011; Fernández, 2012).

Finalmente, en 2003 se aprobó el estándar UNE 150301: Gestión ambiental del proceso de diseño y desarrollo. Ecodiseño, primera estándar certificable sobre esta materia, en la que se describen los requisitos del sistema y compatible con otros sistemas de gestión, de calidad y ambiental (ISO, 2015a; ISO 2015b), utilizados habitualmente por las empresas (AENOR, 2003).

La implantación de la estándar UNE 150301 evolucionó de forma satisfactoria, lo cual animó en ISO a crear un grupo de trabajo en el año 2008, el ISO/TC 207/SC 1/WG 4, promovido por AENOR, que iniciase el proceso de creación de la estándar internacional de ecodiseño basada en la UNE 150301 (Alonso, 2011), la estándar ISO 14006, publicada en julio de 2011.

Mientras que la estándar ISO 14001 se centra en la mejora de los impactos ambientales generados en la etapa de producción, la ISO 14006 va más allá al trasladar el estudio de los distintos aspectos ambientales afectados y el análisis de su impacto a todas las etapas del ciclo de vida del producto, en línea con el Pensamiento de Ciclo de Vida (Life Cycle Thinking) que impera en la Política de Productos Integrada de la Comisión Europea (ISO, 2011a; Comisión Europea, 2001).

En sus primeros años de andadura el estándar no ha tenido una difusión tan importante como otros estándares aprobados en el mismo periodo en el sector industrial (Landeta et al., 2016) como el estándar ISO 50001 (ISO, 2011b) ya que en este sector solamente tenían el certificado 55 empresas en España a finales de 2014 pero proporcionalmente la difusión ha sido muy importante entre las empresas cooperativas industriales vascas, entre las que encontramos 7 empresas que han adoptado y certificado su sistema de gestión conforme al estándar ISO 14006.

Teniendo en cuenta estos antecedentes, el objetivo de este artículo es conocer cómo ha sido el proceso de adopción del estándar de ecodiseño ISO 14006 en las empresas cooperativas del sector de bienes de equipo. Con este objetivo, tras esta introducción se expone el diseño metodológico de la investigación. A continuación, el capítulo 3 está dedicado a analizar los resultados del estudio desarrollado y por último, en las secciones 5 y 6 se muestran las conclusiones y las referencias bibliográficas utilizadas. 


\section{Metodología}

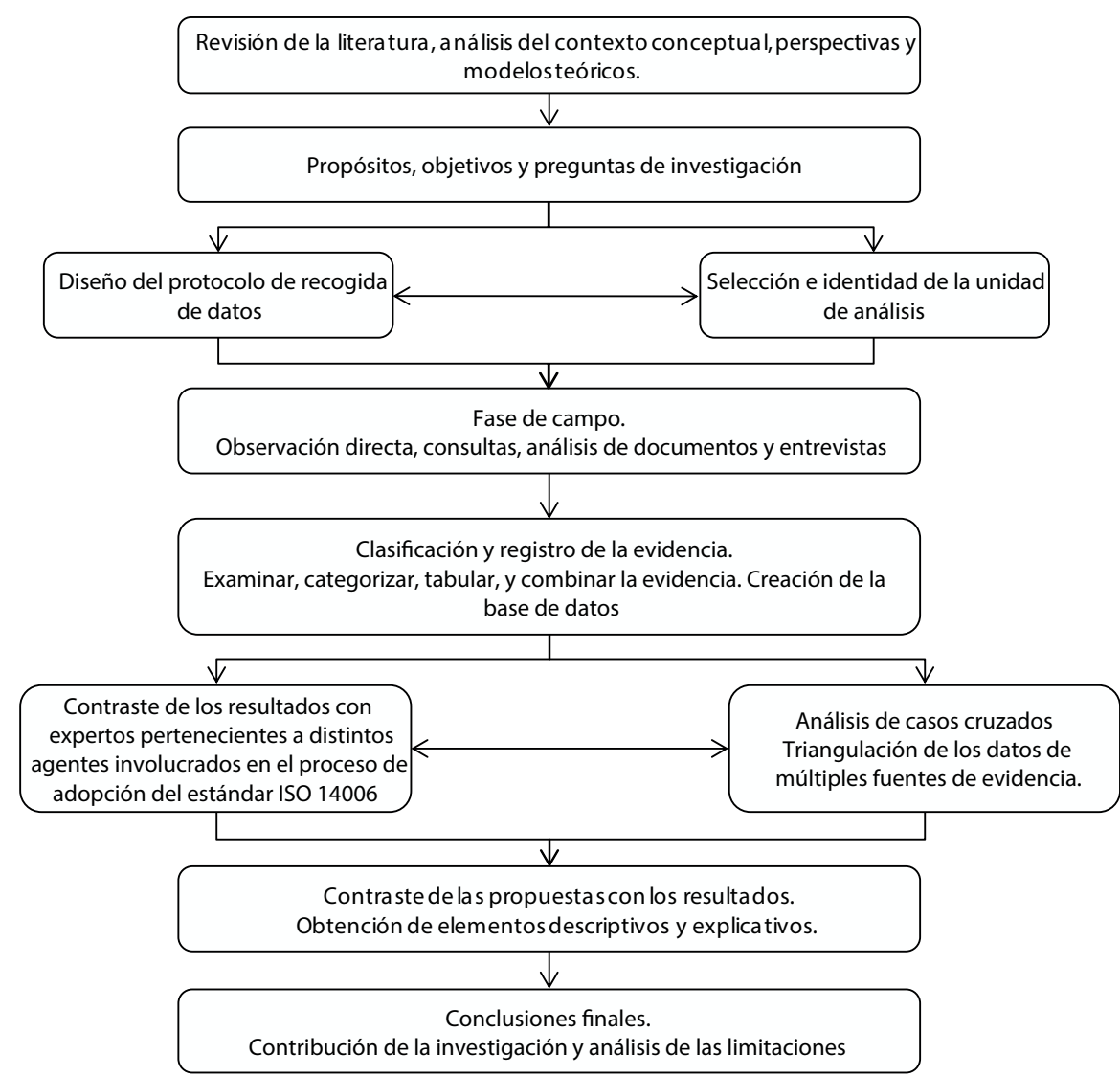

Fuente: elaboración propia.

\section{Figura 1}

Diseño metodológico de la investigación.

Para el desarrollo de este estudio, se ha seleccionado un estudio de casos desarrollado en colaboración con las cooperativas Soraluce y Orona. Esta metodología fue seleccionada porque se adecúa bien al estudio de fenómenos complejos como los procesos de adopción y certificación de estándares de gestión por parte de las organizaciones. En una investigación de naturaleza descriptiva, principalmente exploratoria, esta metodología 
permite profundizar más en el conocimiento del proceso y obtener una mejor comprensión de su naturaleza (Eisenhardt, 1989; Yin, 2009).

Siguiendo el esquema de la figura 1, el estudio se desarrolló entre enero de 2009 y mayo de 2014 y consistió en una secuencia de entrevistas en profundidad semi-estructuradas con directivos siguiendo un guión semiflexible con el mismo número de preguntas y con un protocolo de evaluación de los casos en función de cada factor, siguiendo las pautas marcadas en la literatura de referencia para garantizar la validez y la fiabilidad de la investigación (Maxwell, 2008; Yin, 2009).

\section{Análisis de casos}

Orona y Soraluce son grandes cooperativas con una fuerte proyección internacional e inmersas en procesos de fortalecimiento de su presencia en los mercados donde operan y de implantación en nuevos mercados. En su estrategia de crecimiento de negocio ambas constataron el creciente interés del mercado por productos ambientalmente más sostenibles y, en especial, de mayor eficiencia energética, en un contexto de fuerte competencia que exigía reducir costes.

En ambos casos, para tratar de satisfacer más y mejor las expectativas de los clientes y adaptarse con mayor rapidez a la cada vez más exigente legislación ambiental, era necesario buscar nuevas herramientas de apoyo a la gestión empresarial. La adopción y la certificación del sistema de gestión de ecodiseño conforme a la ISO 14006 fue una apuesta estratégica, fuertemente respaldada por la Dirección, que aún hoy sostienen como acertada.

A continuación, en la tabla 1 se presenta un resumen de aspectos que han influido de forma importante en las motivaciones que han tenido estas empresas para adoptar el estándar.

\section{Tabla 1}

Aspectos que han influido de forma crítica en que la empresa para la adopción y certificación de su sistema de gestión conforme a la ISO 14006.

\begin{tabular}{cll}
\hline \multicolumn{1}{c}{ Aspecto } & \multicolumn{1}{c}{ Soraluce, S.Coop. } & \multicolumn{1}{c}{ Orona, S.Coop. } \\
\hline Clientes & Términos como sostenibilidad, & Creciente interés del mercado por \\
& conciencia ambiental, producto & ascensores energéticamente más \\
& ecológico... valores en alza en- & eficientes y más silenciosos. \\
& tre los clientes de máquina-he- & Tratar de satisfacer más y mejor \\
& ramienta. & las expectativas de los clientes. \\
\hline
\end{tabular}




\begin{tabular}{|c|c|c|}
\hline Aspecto & Soraluce, S.Coop. & Orona, S.Coop. \\
\hline $\begin{array}{l}\text { Imagen de pro- } \\
\text { ducto/servicio }\end{array}$ & $\begin{array}{l}\text { Reducir el consumo de energía } \\
\text { en la fase de uso, las dimensio- } \\
\text { nes de las máquinas y el con- } \\
\text { sumo de consumibles para re- } \\
\text { forzar la imagen de calidad y } \\
\text { eficiencia, y mejorar comporta- } \\
\text { miento ambiental. }\end{array}$ & $\begin{array}{l}\text { Mejora de la imagen del pro- } \\
\text { ducto }\end{array}$ \\
\hline $\begin{array}{l}\text { Imagen de em- } \\
\text { presa }\end{array}$ & $\begin{array}{l}\text { Necesidad de diferenciación de } \\
\text { los competidores. }\end{array}$ & $\begin{array}{l}\text { Mejora de la imagen frente a } \\
\text { competidores. }\end{array}$ \\
\hline $\begin{array}{l}\text { Acceder a nuevos } \\
\text { mercados }\end{array}$ & $\begin{array}{l}\text { Nuevo enfoque de empresa com- } \\
\text { prometida con la sostenibilidad } \\
\text { promovido por asociaciones del } \\
\text { sector (AFM, INVEMA...). Ob- } \\
\text { jetivo: sectores donde los nive- } \\
\text { les de exigencia ambiental de los } \\
\text { proveedores sean elevados. }\end{array}$ & $\begin{array}{l}\text { Mejorar la eficiencia energética de } \\
\text { sus productos de acuerdo con la } \\
\text { demanda de los mercados. Obje- } \\
\text { tivo: máxima calificación energé- } \\
\text { tica (A) de la certificación VDI }{ }^{1} \text {. }\end{array}$ \\
\hline $\begin{array}{l}\text { Acciones de las } \\
\text { Administracio- } \\
\text { nes Públicas }\end{array}$ & $\begin{array}{l}\text { Estudio específico promovido } \\
\text { por Ihobe sobre la aplicación } \\
\text { del ecodiseńo de un producto. }\end{array}$ & $\begin{array}{l}\text { «Servicio Producto Más Ambien- } \\
\text { tal» de Ihobe. }\end{array}$ \\
\hline $\begin{array}{l}\text { Mejora del im- } \\
\text { pacto medioam- } \\
\text { biental de pro- } \\
\text { ductos/servicios }\end{array}$ & $\begin{array}{l}\text { El ecodiseño visto como una } \\
\text { herramienta para reducir el con- } \\
\text { sumo eléctrico de las máquinas } \\
\text { en su fase de uso, mejora de la } \\
\text { funcionalidad, e incluso reducir } \\
\text { el coste del producto. }\end{array}$ & $\begin{array}{l}\text { Optimización de aspectos relacio- } \\
\text { nados con la ecoeficiencia, la segu- } \\
\text { ridad y el confort de los productos. } \\
\text { Apuesta por desarrollar sistemas de } \\
\text { elevación ambiental, social, energé- } \\
\text { tico y económicamente sostenibles. }\end{array}$ \\
\hline $\begin{array}{l}\text { Concienciación } \\
\text { medioambiental }\end{array}$ & $\begin{array}{l}\text { Compromiso con los valores y } \\
\text { principios ecológicos, económi- } \\
\text { cos y sociales del desarrollo soste- } \\
\text { nible mediante la fabricación de } \\
\text { productos, tecnologías y proce- } \\
\text { sos más sostenibles. }\end{array}$ & $\begin{array}{l}\text { Respeto por el medio ambiente. } \\
\text { Orona IDeO: 1er centro europeo } \\
\text { I+D+i para una movilidad urbana } \\
\text { sostenible y autosuficiente. }\end{array}$ \\
\hline
\end{tabular}

Fuente: elaborado a partir de los datos proporcionados por Soraluce y Orona.

En el proceso de adopción del estándar ISO 14006, la experiencia previa en sistemas de gestión de las dos empresas fue un elemento facilitador clave

1 VDI4707 es una medición estándar sobre consumo energético en ascensores establecida en marzo de 2009 por la Asociación de Ingenieros Alemanes. 
en ambos casos. Concretamente, Soraluce contaba con un sistema de gestión integrado certificado conforme a la ISO 9001, ISO 14001 y OHSAS 18001 (OHSAS, 2007), e incluso había obtenido sido premiado con la Q de oro al obtener más de 500 puntos en la evaluación realizada por Euskalit conforme a los criterios del modelo EFQM.

Por otra parte, Orona contaba con gran experiencia en la utilización de sistemas de gestión basados en estándares internacionales, ya que ha sido una de las primeras empresas vascas en certificarse conforme a la ISO 9001 y la ISO 14001. Al contar con un manual de gestión y unos procedimientos desarrollados, tan sólo tuvieron que incluir los nuevos requisitos exigidos por el estándar ISO 14006.

En cualquier caso, las empresas entrevistadas señalan otros factores clave para el desarrollo adecuado de todo el proceso. Así, es muy importante la implicación de la Dirección de la empresa, que debe disponer de los instrumentos y las personas para llevar a buen puerto el proceso de adopción del estándar, elegir adecuadamente los responsables del mismo y proporcionar las ayudas externas necesarias con experiencia contrastada para la preparación y asesoramiento en dicho proceso.

Los encargados del proceso deben, a su vez, realizar y aprobar la planificación anual de ecodiseño, asesorar en la generación, selección y evaluación de ideas de mejora tanto desde el punto de vista técnico como medioambiental, y participar en la búsqueda de alternativas a materiales, componentes, proveedores, procesos de fabricación...

En este punto, las empresas entrevistadas manifiestan una carencia en la formación y conocimientos en ecodiseño de los técnicos y jefes del área de diseño y desarrollo de producto, lo cual dificulta la adopción de criterios ambientales en la fase de diseńo y desarrollo, de acuerdo con el pensamiento de ciclo de vida (Life Cycle Thinking). El tratamiento que debe tener la variable ambiental en dicha fase es desconocido para una mayoría del equipo de técnicos, aún a pesar de que en dicha fase tengan origen el $80 \%$ de los impactos ambientales del producto. A este respecto, el mercado laboral tampoco ha dado respuesta a la demanda de profesionales con dominio en ecodiseño de las empresas.

Por ello es preciso que el personal técnico y responsables del diseño y desarrollo de producto reciban charlas informativas y cursos de formación específicos sobre los procesos de gestión del ecodiseño y sobre la utilización e integración de herramientas para identificación, cálculo y evaluación de impactos ambientales como, por ejemplo, aquellas basadas en la metodología de Análisis de Ciclo de Vida (ACV). En un principio, durante el proceso de adopción y certificación la formación la diseñan y la imparten las propias consultoras que han acompañado a las empresas en todo el proceso de adopción y certificación conforme al estándar ISO 14006. Después, las acciones formativas son impartidas principalmente por personal experto propio, apo- 
yado puntualmente de personal externo (auditores, consultores y organismos públicos de medioambiente, principalmente Ihobe).

Sin embargo, según precisan las empresas, en determinadas ocasiones la formación recibida adolecía de respuestas a problemas de experiencias prácticas de los técnicos y a dificultades en el manejo e integración en sus procesos de herramientas específicas de ecodiseño.

A este respecto, una vez efectuado el desarrollo documental en el siguiente paso dado por ambas empresas, Orona y Soraluce, ambas empresas, se encontraron con una de sus principales dificultades de todo el proceso fue realizar el diagnóstico ambiental de la referencia de comparación del producto a ecodiseñar. Concretamente, Soraluce se encontró con problemas para la recopilación e inventariado del ciclo de vida del producto, debido especialmente a la gran diversidad de materiales, procesos y componentes asociados. Además, las herramientas basadas en ACV les resultaban muy complejas y con arquitecturas cerradas difíciles de adaptar e integrar a sus necesidades, por lo que optaron por desarrollar su propio software. Por su parte, Orona trabajó en completar la base de datos de Ecoinvent de Simapro, un software comercial, para adaptarlo a su problemática.

Una vez solucionados estos problemas, se desarrolló la identificación y evaluación de aspectos e impactos ambientales los productos, cuyo perfil por fases del ciclo de vida del producto en términos porcentuales se muestra en el gráfico 1 .

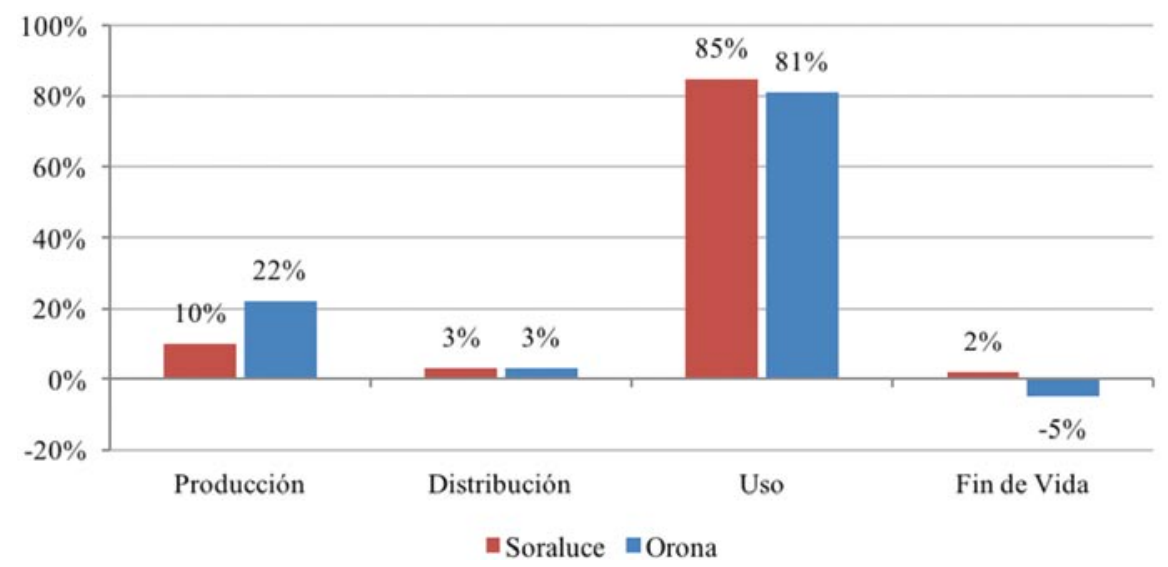

Fuente: elaborado a partir de los datos proporcionados por Soraluce y Orona.

\section{Gráfico 1}

Perfil ambiental del ciclo de vida la fresadora $T R$ de Soraluce y el ascensor $M 322$ de Orona. 
Como se observa en el gráfico, los perfiles ambientales son similares entre la fresadora de Soraluce y el ascensor de Orona. Ambos precisan de energía eléctrica para su utilización y es por ello que en ambos casos más del $80 \%$ de su impacto ambiental se produce en la fase de uso.

A partir de este análisis previo basado en indicadores ambientales, en ambos casos se estableció una estrategia para reducir el impacto ambiental de sus productos. Esta estrategia se resume en la tabla 2.

Tabla 2

Principales estrategias y medidas de ecodiseño consideradas para la mejora ambiental de la fresadora $T R$ de Soraluce y el ascensor M322 de Orona

\begin{tabular}{|c|c|c|c|}
\hline Fase & Estrategia & Caso & Medida aplicada \\
\hline \multirow[t]{2}{*}{$\begin{array}{l}\text { Obtención de } \\
\text { materiales y } \\
\text { componentes }\end{array}$} & \multirow[t]{2}{*}{$\begin{array}{l}\text { Reducir el uso de } \\
\text { material }\end{array}$} & Soraluce & $\begin{array}{l}\text { Disminución de la cantidad de } \\
\text { materia prima necesaria en la fa- } \\
\text { bricación de las ruedas dentadas } \\
\text { de la multiplicadora. } \\
\text { Reconfiguración de la estructura } \\
\text { original de la bancada. }\end{array}$ \\
\hline & & Orona & $\begin{array}{l}\text { Rediseño de la unidad motriz, } \\
\text { estructura, cabina, contrapeso y } \\
\text { sistemas de guiado }\end{array}$ \\
\hline \multirow[b]{2}{*}{ Producción } & \multirow[b]{2}{*}{$\begin{array}{l}\text { Reducción de } \\
\text { substancias con- } \\
\text { taminantes }\end{array}$} & Soraluce & $\begin{array}{l}\text { Utilizar pinturas sin disolventes, } \\
\text { libre de } \mathrm{COVs}^{2} \text {. }\end{array}$ \\
\hline & & Orona & $\begin{array}{l}\text { Eliminar operaciones con oxi- } \\
\text { corte. } \\
\text { Utilización de equipos sin nece- } \\
\text { sidades de lubricación. }\end{array}$ \\
\hline \multirow{2}{*}{$\begin{array}{l}\text { Transporte y } \\
\text { distribución }\end{array}$} & \multirow{2}{*}{$\begin{array}{l}\text { Seleccionar for- } \\
\text { mas de distribu- } \\
\text { ción ambiental- } \\
\text { mente eficientes }\end{array}$} & Soraluce & - \\
\hline & & Orona & $\begin{array}{l}\text { Reducción en los pesos y huecos } \\
\text { del embalaje. }\end{array}$ \\
\hline
\end{tabular}

2 Compuestos Orgánicos Volátiles. 


\begin{tabular}{|c|c|c|c|}
\hline Fase & Estrategia & Caso & Medida aplicada \\
\hline \multirow{6}{*}{ Uso } & \multirow{2}{*}{$\begin{array}{l}\text { Reducir las nece- } \\
\text { sidades de man- } \\
\text { tenimiento }\end{array}$} & Soraluce & $\begin{array}{l}\text { Sustitución del aceite de lubri- } \\
\text { ficación por grasa mediante un } \\
\text { sistema de engrase automático } \\
\text { centralizado. }\end{array}$ \\
\hline & & Orona & $\begin{array}{l}\text { Utilización de equipos sin nece- } \\
\text { sidades de lubricación }\end{array}$ \\
\hline & \multirow{4}{*}{$\begin{array}{l}\text { Reducir el con- } \\
\text { sumo energético }\end{array}$} & Soraluce & $\begin{array}{l}\text { Empleo de accionamientos con } \\
\text { una mayor eficiencia energética. }\end{array}$ \\
\hline & & & $\begin{array}{l}\text { Introducción de un sistema de } \\
\text { regeneración, aprovechando la } \\
\text { energía generada durante el fre- } \\
\text { nado. }\end{array}$ \\
\hline & & Orona & $\begin{array}{l}\text { Rediseño del contrapeso, el con- } \\
\text { trol del freno motor y el varia- } \\
\text { dor. }\end{array}$ \\
\hline & & & $\begin{array}{l}\text { Utilización de sistema de alum- } \\
\text { brado de bajo consumo. Ela- } \\
\text { boración de un manual de re- } \\
\text { ciclaje para el ascensor con las } \\
\text { tecnologías actualmente dispo- } \\
\text { nibles. }\end{array}$ \\
\hline \multirow[b]{2}{*}{ Fin de vida } & \multirow{2}{*}{$\begin{array}{l}\text { Optimizar el ci- } \\
\text { clo de vida }\end{array}$} & Soraluce & - \\
\hline & & Orona & $\begin{array}{l}\text { Reducción en los pesos y huecos } \\
\text { del embalaje. }\end{array}$ \\
\hline
\end{tabular}

Fuente: elaborado a partir de los datos proporcionados por Soraluce y Orona.

En ambos casos, las medidas más importantes se han centrado en reducir el consumo eléctrico de la fase de uso. Asimismo se ha trabajado en la reducción del consumo de materiales en el diseño y en la utilización de materiales reciclables, lo que redunda en la mejora del comportamiento de ambos productos ecodiseñados en la fase de fin de vida del producto.

No obstante, reducir el uso de material y/o emplear materiales reciclables, exigió extender la estrategia de ecodiseño a la cadena de suministro para la mejora del desempeño ambiental de productos, aspecto que ambos casos no ha estado exento de dificultades principalmente debidas a la falta de conocimiento y formación de los proveedores. 
90 B. LANDETA-MANZANO, G. ARANA-LANDÍN, P. RUÍZ DE ARBULO Y P. DÍAZ DE BASURTO

En cualquier caso, una vez aplicadas las medidas de mejora ambiental acordadas se volvieron a analizar los indicadores y comparar con los indicadores del producto de referencia.

En la tabla 3 se muestra un resumen de los principales indicadores ambientales afectados por la adopción de las medidas de mejora y en el gráfico 2 se presenta la mejora porcentual de cada uno de los indicadores.

\section{Tabla 3}

Indicadores de impacto ambiental de los modelos inicial y ecodiseñados.

\begin{tabular}{|c|c|c|c|c|}
\hline \multirow{3}{*}{ CATEGORIA DE IMPACTO } & \multirow{2}{*}{\multicolumn{2}{|c|}{$\frac{\text { Fresadora TR }}{\text { TOTAL }}$}} & \multirow{2}{*}{\multicolumn{2}{|c|}{$\frac{\text { Ascensor M322 }}{\text { TOTAL }}$}} \\
\hline & & & & \\
\hline & Inicial & Final & Inicial & Final \\
\hline Energía bruta (MJ primario) & 66.739 .611 & 55.654 .856 & 1.530 .000 & 1.100 .000 \\
\hline Electricidad (MJ primario) & 65.646 .291 & 54.704 .625 & 1.390 .000 & 1.000 .000 \\
\hline Poder calorífico neto (MJ primario) & 274.528 & 217.768 & 3.820 & 2.410 \\
\hline Agua de proceso (ltr. Agua) & 4.437 .905 & 3.700 .981 & 121.000 & 81.200 \\
\hline Agua de refrigeración (ltr. Agua) & 174.952 .478 & 145.797 .511 & 3.650 .000 & 2.640 .000 \\
\hline Residuos peligrosos (g residuos) & 1.620 .459 & 1.368 .349 & 88.100 & 75.700 \\
\hline Residuos no peligrosos (g residuos) & 90.857 .958 & 77.342 .927 & 6.120 .000 & 3.940 .000 \\
\hline Calentamiento global (kg CO2 eq.) & 2.912 .902 & 2.432 .125 & 71.600 & 51.100 \\
\hline Acidificación (g SO2 eq.) & 17.114 .485 & 14.279 .329 & 411.000 & 291.000 \\
\hline COVs (g NMVOCs) & 127.839 & 103.067 & 1.580 & 1.160 \\
\hline COPs (ng TCDD eq.) & 616.264 & 532.690 & 60.200 & 37.700 \\
\hline Metales pesados aire (mg Ni eq.) & 1.269 .029 & 1.064 .532 & 100.000 & 60.300 \\
\hline PAHs (mg Ni eq.) & 163.064 & 136.797 & 13.900 & 6.100 \\
\hline Partículas (g partículas & 1.386 .914 & 1.293 .542 & 125.000 & 113.000 \\
\hline Metales pesados agua (mg Hg/20 eq.) & 1.126 .467 & 914.668 & 52.200 & 28.800 \\
\hline Eutrofización (mg pO4 eq.) & 10.764 .340 & 8.800 .713 & 1.190 .000 & 668.000 \\
\hline
\end{tabular}

Fuente: elaborado a partir de los datos proporcionados por Soraluce y Orona. 


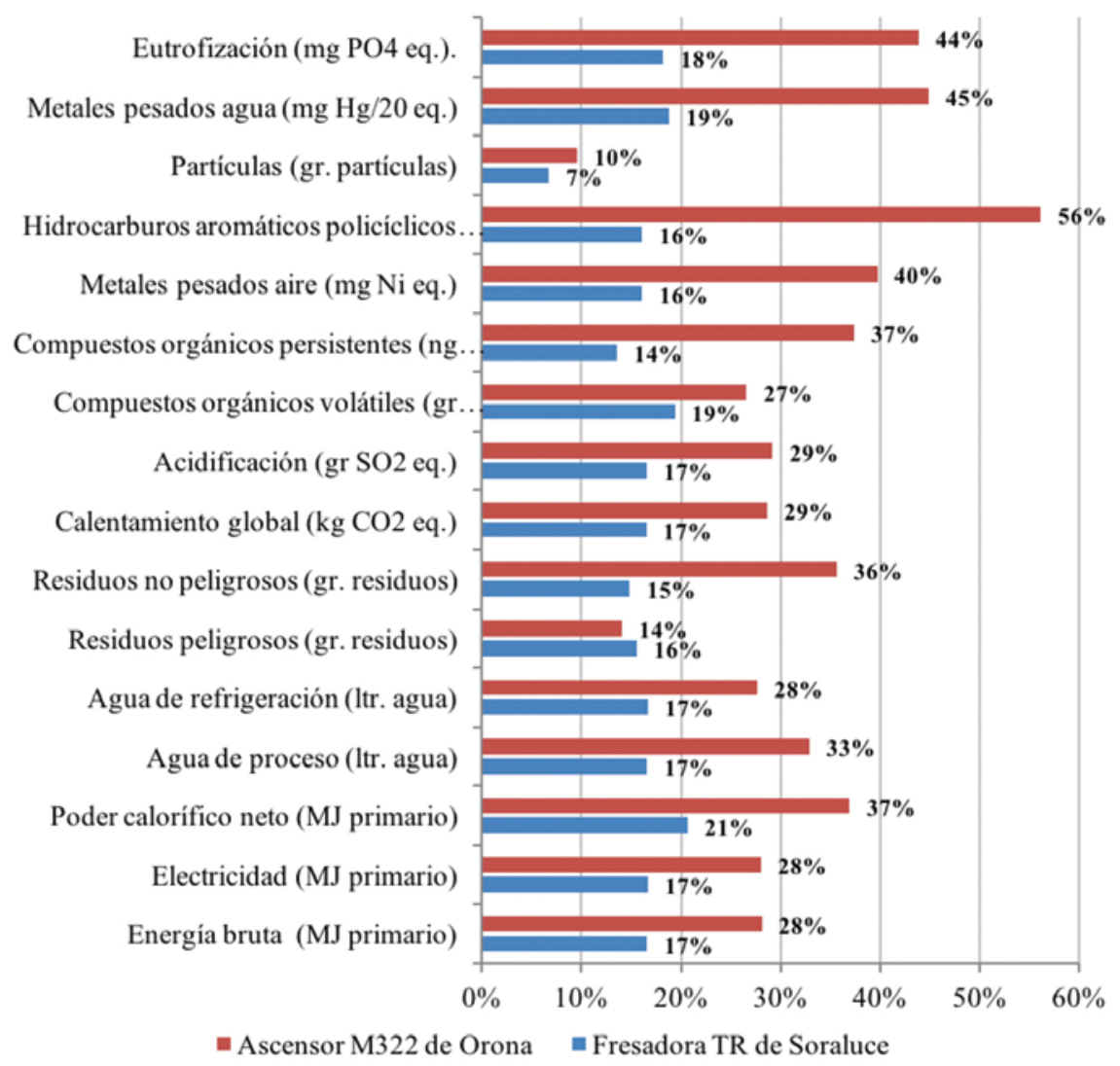

Nota: Las cifras positivas indican una mejora del desempeño ambiental.

Fuente: elaborado a partir de los datos proporcionados por Soraluce y Orona.

\section{Gráfico 2}

Resultados de desempeño ambiental conseguidos en todo el ciclo de vida la fresadora $T R$ de Soraluce y el ascensor M322 de Orona.

A continuación, en la tabla 4 se muestra, se presenta un análisis comparativo aplicando el procedimiento analítico de identificación basado $\mathrm{Mi}$ les y Huberman (1994), de los resultados de la adopción y certificación conforme al estándar ISO 14006 obtenidos por las empresas. En la tabla se muestran los textos descriptivos de los resultados obtenidos de la exploración a través de las diversas fuentes de las evidencias encontradas de los casos objeto de estudio. 


\section{Tabla 4}

Síntesis de los resultados del estudio de casos de la agrupación sectorial de bienes de equipo

\begin{tabular}{|c|c|c|}
\hline Aspecto & Soraluce, S.Coop. & Orona, S.Coop. \\
\hline $\begin{array}{l}\text { Mejora de los re- } \\
\text { sultados económi- } \\
\text { cos }\end{array}$ & $\begin{array}{l}\text { En mercados de referencia exi- } \\
\text { gentes como el alemán, el eco- } \\
\text { diseño les ha ayudado a mante- } \\
\text { nerse. } \\
\text { Coste de máquina ligeramente } \\
\text { superior }(+0,1-0,2 \%) \\
\text { El cliente valora las mejoras, pero } \\
\text { no implica que vaya a pagar más } \\
\text { por una máquina ecodiseñada. }\end{array}$ & $\begin{array}{l}\text { Éxito de ventas del modelo } \\
\text { ecodiseñado (Orona } 3 \mathrm{G} \\
1015 \text { ) } \\
\text { Mejora del coste del pro- } \\
\text { ducto. }\end{array}$ \\
\hline $\begin{array}{l}\text { Aumento del va- } \\
\text { lor ańadido }\end{array}$ & $\begin{array}{l}\text { Herramienta de innovación: } \\
\text { Mejoras introducidas a través } \\
\text { del ecodiseńo en factores clave } \\
\text { del producto como la funciona- } \\
\text { lidad, fiabilidad o durabilidad, } \\
\text { eficiencia energética y reducción } \\
\text { de residuos generados. }\end{array}$ & $\begin{array}{l}\text { Mejora de aspectos que el } \\
\text { cliente valora en su compra } \\
\text { como el coste del producto, } \\
\text { eficiencia energética y la ca- } \\
\text { lidad. }\end{array}$ \\
\hline $\begin{array}{l}\text { Mejora de su ima- } \\
\text { gen de producto/ } \\
\text { servicio. }\end{array}$ & $\begin{array}{l}\text { El cliente valora de valora las } \\
\text { mejoras. }\end{array}$ & $\begin{array}{l}\text { La certificación refuerza la } \\
\text { imagen de marca. }\end{array}$ \\
\hline $\begin{array}{l}\text { Mejora de su ima- } \\
\text { gen de empresa. }\end{array}$ & $\begin{array}{l}\text { Proyección de Soraluce como } \\
\text { fabricante innovador y sensibili- } \\
\text { zado con el medio ambiente: «el } \\
\text { ecodiseño nos hace más compe- } \\
\text { titivos comercialmente y distin- } \\
\text { guirnos de otros competidores» }\end{array}$ & $\begin{array}{l}\text { Supone un diferencial com- } \\
\text { petitivo y estratégico. }\end{array}$ \\
\hline $\begin{array}{l}\text { Anticipación en el } \\
\text { cumplimiento de } \\
\text { la legislación. }\end{array}$ & $\begin{array}{l}\text { Anticipación al cumplimiento } \\
\text { de las futuras exigencias lega- } \\
\text { les, p.ej.: Directiva } 2009 / 125 / \\
\text { CE (ErP) }\end{array}$ & $\begin{array}{l}\text { Anticipación al cumpli- } \\
\text { miento de las futuras exigen- } \\
\text { cias legales, p.ej.: Directiva } \\
\text { 2009/125/CE (ErP) }\end{array}$ \\
\hline $\begin{array}{l}\text { Satisfacción con } \\
\text { los resultados }\end{array}$ & $\begin{array}{l}\text { Satisfechos con la adopción y } \\
\text { certificación de ecodiseño. } \\
\text { La ISO } 14006 \text { es demasiado } \\
\text { flexible en cuanto al nivel de exi- } \\
\text { gencia. }\end{array}$ & $\begin{array}{l}\text { Satisfechos con la adopción } \\
\text { del estándar de ecodiseño, } \\
\text { consideran que les han ayu- } \\
\text { dado a mejorar el producto. }\end{array}$ \\
\hline
\end{tabular}

Fuente: elaboración propia. 


\section{Conclusiones}

El creciente interés del mercado por productos ambientalmente más sostenibles, tratar de satisfacer más y mejor las expectativas de los clientes, adaptarse con mayor rapidez a la cada vez más exigente legislación ambiental y, en especial, crear productos energéticamente más eficientes y paralelamente reducir de los costes de producto, ha llevado a estas cooperativas a buscar nuevas herramientas de apoyo a la gestión empresarial. En este sentido, Orona y Soraluce consideran que el estándar de ecodiseño ISO 14006 es una herramienta adecuada para aquellas empresas que muestren una disposición permanente para innovar. Otro motivo para adoptar el estándar que mencionan es la determinación firme de trabajar por el desarrollo sostenible de su actividad.

En el proceso de adopción y certificación conforme al estándar, la experiencia en la adopción de sistemas de gestión es un elemento que facilita en gran medida la adopción del ecodiseño. Sin embargo, este proceso no ha estado exento de dificultades, destacando entre ellas, la falta de dominio de las propias empresas en materia de ecodiseño, que ha creado situaciones de duda y desconocimiento que dificultan el proceso.

El uso de herramientas de ACV ha supuesto un descubrimiento para los técnicos del área de diseño y desarrollo de producto, pues, además de permitirles centrarse en los puntos débiles del producto, ha permitido mejorar la perspectiva integral del producto y comprobar la influencia ambiental de sus decisiones en todo el ciclo de vida del mismo.

Sin embargo, los técnicos van más allá y demandan una herramienta de ACV integrada con las herramientas habituales de trabajo propias del área e, incluso, con gestores de bases de datos empleados en la empresa para la determinación y control de costes, con el fin de poder conocer en todo momento el coste asociado a un impacto ambiental determinado y así poder actuar para tratar de reducirlo.

En todo caso, la implantación de las directrices del estándar de ecodiseño en el sistema de gestión de la empresa ha posibilitado un mayor poder de innovación en el diseño y desarrollo de productos y procesos asociados (cadena de suministro, fabricación, transporte, instalación, operación y mantenimiento...). Las mejoras introducidas a través del ecodiseño en factores clave del producto (la calidad, la eficiencia energética en la fase de uso o la generación de menos residuos en el fin de vida...) le dan un valor añadido al producto y proyectan una imagen al cliente de empresa innovadora y sensibilizada con el medio ambiente.

En general, Orona y Soraluce consideran el ecodiseño como un elemento diferenciador en el mercado que el cliente valora. No obstante, una consecuencia directa de la aplicación del ecodiseño es un ligero aumento 
de los costes de la máquina, debido principalmente al empleo de equipos más eficientes pero más caros. Sin embargo, en la mayor parte de los casos el cliente no está dispuesto a pagar más por un producto ecodiseñado, aunque en la fase de uso, los ahorros principalmente energéticos compensen con creces el precio de adquisición del producto.

A este respecto, las empresas del sector señalan que es preciso que las administraciones públicas promuevan medidas que favorezcan el desarrollo de mercados «verdes». Entre otras medidas proponen el desarrollo de la legislación en materia ambiental. Se debe primar al producto más eficiente ambientalmente o gravar el que peor comportamiento presente. No hacerlo, a su juicio, crearía una situación de competencia desleal. Muchas empresas que no realizan esfuerzos en innovación de producto fabrican productos menos eficientes y con mayor impacto ambiental pero a menor coste. Y el coste del producto es, precisamente, el parámetro de compra más determinante de un cliente en la mayoría de mercados actualmente.

\section{Agradecimientos}

Este artículo es un resultado del Grupo de Investigación del Sistema Universitario Vasco IT763-13 / GIC12-158 financiado por el Gobierno Vasco. Los autores desean mostrar especialmente su agradecimiento a los directivos y técnicos de las cooperativas de Orona y Soraluce, así como, a los miembros del grupo de expertos que han colaborado en las distintas fases de esta investigación.

\section{Referencias bibliográficas}

AENOR (2003) UNE150301: 2003. Gestión ambiental del proceso de diseño y desarrollo. Ecodiseño, Madrid.

Alonso, C (2011) La Norma UNE-EN ISO 14006:2011: Directrices para la incorporación del ecodiseño en un sistema de gestión ambiental, Bilbao Ecodesign Meeting 2011, Bilbao, España.

Arana-Landín G, Heras-Saizarbitoria I, Cilleruelo-Carrasco E (2012) A case study of the adoption of a reference standard for ISO 14006 in the lift industry. Clean Technol Environ Policy 14 (4):641-649.

Arana-Landín G, Heras-Saizarbitoria I (2011) Paving the way for the ISO 14006 ecodesign standard: an exploratory study in Spanish companies. J Cleaner Prod 19(9):1007-1015.

Comisión Europea (2011) Informe de 23 de noviembre de 2001 sobre el Libro Verde de la Comisión sobre la política de productos integrada. Comisión de Medio Ambiente, Salud Pública y Política del Consumidor, Bruselas. 
Eisenhardt KM (1989) Building theories from case study research. Acad Manage Rev 14(4):532-550.

Fernández JM (2012) Ecodiseño en los sistemas de gestión ambiental según UNE-EN ISO 14006:2011. Aplicación a una empresa de base tecnológica. Dyna 88(5):514-517.

ISO (2015a) ISO 9001:2015. Quality management systems. Requirements, Geneva.

ISO (2015b) ISO 14001:2015. Environmental management systems. Requirements with guidance for use, Geneva.

ISO (2011a) ISO 14006:2011. Environmental management systems. Guidelines for incorporating ecodesign, Geneva.

ISO (2011b) ISO 50001:2011. Energy management systems. Requirements with guidance for use, Geneva.

Landeta, B, Arana-Landín, G, RuizdeArbulo, P, DíazdeBasurto, P (2016) Longitudinal Analysis of the Eco-Design Management Standardization Process in Furniture Companies. Journal of Industrial Ecology.

Landeta B, Arana-Landín G, Ruíz de Arbulo P, Díaz de Basurto P (2015) Sustainability through Eco-Design: Shedding light on the adoption of the ISO 14006 Standard. In Sustainable Operations Management (pp. 163-181). Springer International Publishing.

Maxwell, JA (2008) Designing a qualitative study. The SAGE handbook of applied social research methods, 2, 214-253.

Miles MB, Huberman AM (1994) Qualitative data analysis: an expanded sourcebook. Sage, California.

Ramos, M. L. P. (2012) Oportunidades para el desarrollo de los fabricantes de bienes de equipo: análisis de la situación y evaluación del sector. Economía industrial, (385), 65-72.

Yin RK (2009) Case study research: design and methods. Sage, California. 